\title{
Erratum to: A Comparative Evaluation of Denoising of Remotely Sensed Images Using Wavelet, Curvelet and Contourlet Transforms
}

\author{
Rizwan Ahmed Ansari ${ }^{1}$. Krishna Mohan Buddhiraju ${ }^{1}$
}

Published online: 2 April 2016

(C) Indian Society of Remote Sensing 2016

Erratum to: J Indian Soc Remote Sens

DOI 10.1007/s12524-016-0552-y

The published online version contains mistake on the second author's name. Correct name should be Krishna Mohan Buddhiraju and not Kirshna Mohan Budhhiraju. Corrected name is provided in the author group section above and affiliation section below.

The online version of the original article can be found at http://dx.doi.org/ 10.1007/s12524-016-0552-y.

Rizwan Ahmed Ansari

rizwan@iitb.ac.in; rizwan.vjti@gmail.com

Krishna Mohan Buddhiraju

bkmohan@csre.iitb.ac.in

1 Satellite Image Processing Lab, Centre of Studies in Resources Engineering (CSRE), Indian Institute of Technology, Bombay, Powai, Mumbai 400076, India 